\title{
AN ENGINEERING APPRAISAL OF POWERED PROSTHESES
}

\author{
T. H. LAMBerT, LoNdon, ENGLAND \\ From the Department of Mechanical Engineering, University College London
}

It is now generally accepted that the application of external power is desirable for bilateral upper limb prostheses. The need for external power increases with the severity of amputation or congenital deformity. It would clearly be over-optimistic to believe that prostheses capable of all the refined movements of the human arm are possible at present: nevertheless the present state of the art both in medicine and engineering does suggest that more versatile prostheses than are at present available could be produced in the near future. These should enable the user to achieve a good deal of independence in the essential daily functions.

Many of the problems involved in the improvement of powered prostheses lie in the no-man's-land between the recognisable frontiers of medicine and engineering. Clearly, advances within the recognised frontiers are of immense value, but the full benefits can te realised quickly only if there is a sufficient body of workers from both disciplines who have ventured into the no-man's-land and who have a fair understanding of each other's problems.

\section{CONTROL SYSTEMS}

Most of the systems now in use are powered pneumatically. A small number of electrically operated devices is becoming available (for example the Russian arm and the rather more refined system developed by Bottomley), but at present these are restricted to the terminal device or hand where relatively low power is required. Higher powered electrical systems and hydraulic systems for artificial limbs present more difficult engineering problems, probably to the encouragement of the development of pneumatic systems.

British pneumatic systems now employ a control valve in which the gas flow increases progressively with valve opening. The valves formerly used had an undesirable characteristic in that minute valve movements produced large changes in gas flow; this offered little possibility of graded control and virtually condemned the user to operate them in an "on-off" manner.

A typical pneumatic system is shown diagrammatically in Figure 1. Here the user or human operator (H.O. in the Figures) causes the valve spindle to be displaced. When considering the prosthesis this command by the patient may be regarded as an input signal to the system. The form of command signal will necessarily depend upon the individual patient. A phocomelic patient may be able to operate the valve spindle directly with a rudimentary digit, whereas an amelic patient may operate the valve through a cable.

If we regard the position of the controlled member as being the output from the system, then the velocity of the member will be a function of the gas flow through the valve which will be related to the input signal. Thus we have a velocity control system in which the speed of movement is related to the command signal. Although the operator may be interested in the velocity of movement the prime object will be to position the prosthesis, and in order to do this the operator must carefully observe the behaviour of the device and modify the input signal accordingly. The human operator is a good controller and, with practice, can make the desired corrections in order to achieve accurate control of position. Is this the best system to use? Experience in other fields suggests that it is not. It is generally accepted that control of position can be effected more accurately and learnt more quickly if the position of the controlled member rather than its velocity is made a function of the input or command signal. How this can be accomplished can be appreciated by considering the way in which the human 
operator controls the system shown in Figure 1. In order to achieve a desired position of the controlled member he must observe its position, compare it with the required value, and operate the valve in such a manner that it reduces the error between the actual and desired position to zero. An essential requirement is that the operator must at all times be informed of the actual position of the controlled member. He will normally gain this information by visual observation. This is often referred to as visual feedback and simply indicates that information regarding the position of the output is conveyed back to the operator by sight.

A system which simplifies the control of the operator is shown in Figure 2. Here the position of the output is fed back by mechanical or electrical means and compared with the desired valve (or input) to generate the error, which is then fed forward to the valve. The comparison element is indicated on the diagram by a circle, and the algebraic signs indicate that the error is determined by input value minus output value. In a well designed system the error will always be small and in the steady state should be zero, so that the output position is now directly related to the input and the operator can learn to associate the value of the command signal with the position of the device. Although visual feedback will still assist the user, the requirements of the physiological computer are eased. The velocity of the output can be controlled by varying the rate at which the input signal is applied.

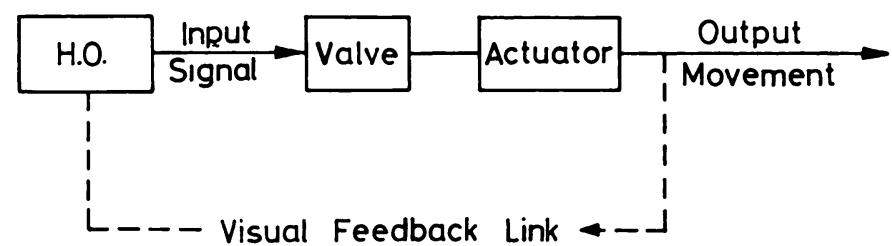

FIG. 1

Velocity control system. H.O. indicates human operator.

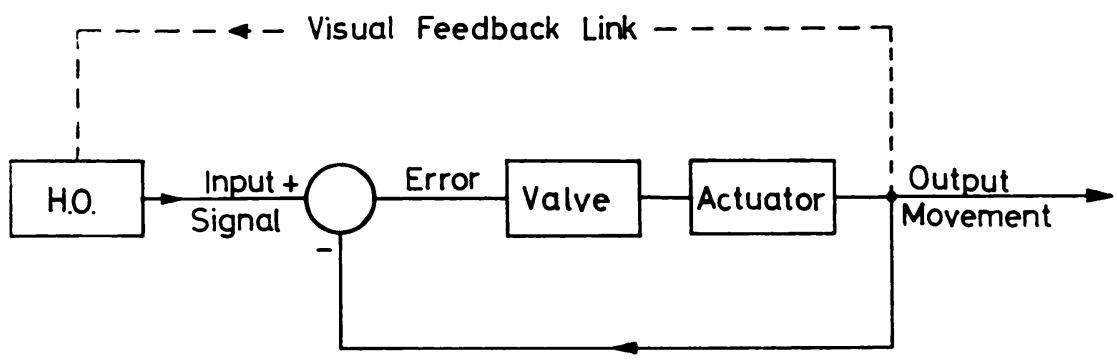

Fig. 2

Position control system. H.O. indicates human operator

The system described above is called a closed loop position control system, the loop being formed by the feedback path between the output and comparison element and the forward error path between the comparison element and the output. At first sight there seems no reason why such a system should not be directly applied to prosthetic use*. There are, however, two problems that must first be overcome. If the normal human arm is held with the forearm horizontal, the tension in the brachialis and biceps muscles supports the weight of the limb and if this is long maintained fatigue and discomfort occur. On the other hand if the weight of the forearm is supported on the arm-rest of a chair, the brachialis and biceps can be relaxed and the posture maintained without discomfort. This would not be so with a closed loop system of position control unless this particular position coincided with the relaxed position

* Since this paper was submitted Dr D. C. Simpson, of the Department of Medical Physics, Royal Infirmary, Edinburgh, has used pneumatic position control systems with thalidomide children at the Princess Margaret Rose Hospital, and he reports success with them. 
of the control site, in which case the extended position of the arm would require a continuous signal from, and consequent fatigue of, the control site. Clearly some means of disengaging the input to the system is required in order to give an infinite variety of relaxed postures.

The second problem is associated with the stability of the closed loop system. The response of a typical closed loop system of position control to a sudden change of input is shown in Figure 3.

In practice the output can never follow the input exactly but this desirable criterion can be approached by increasing the gain of the system. Unfortunately this will also increase

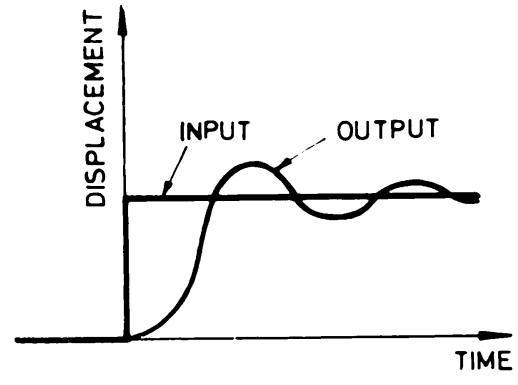

FiG. 3

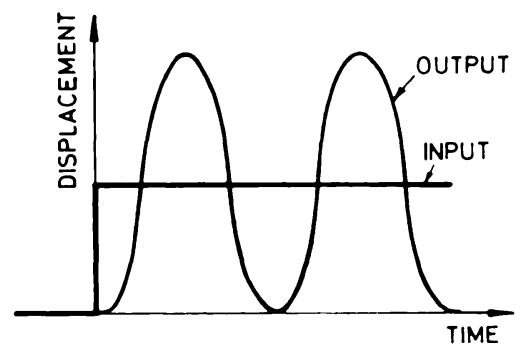

Fig. 4

Stable and unstable responses of a closed-loop control system.

the oscillatory nature of the response and may cause this to persist indefinitely (Fig. 4). Stability can be regained by introducing dissipative damping at the output, but this is usually costly both in weight and power, and a more elegant solution is achieved either by velocity feedback or by some form of derivative action introduced into the forward error path.

Both problems can be overcome in wholly pneumatic systems but more flexibility in terms of variations in gain and damping can be achieved with an electro-pneumatic system. It is perhaps worth mentioning that the pneumatic servo-mechanism is a significantly non-linear device whose analysis is quite a formidable task. Approximate theories are available but engineers use these with a great deal of caution.

\section{MULTIPLE CONTROL SYSTEMS}

The human arm is a versatile instrument which enables the terminal device to be positioned anywhere in three-dimensional space. In addition the hand can be orientated at a variety of angles at this position. Although one cannot at present hope to reproduce the complete flexibility of the arm, it seems probable that a good degree of independence could be achieved by the patient if in addition to prehension three or four degrees of freedom were introduced between the trunk and the terminal device. The best movements to choose for this may be the subject of some discussion but one solution could be: wrist rotation, elbow flexion, some form of humeral rotation together with rotation about another axis at the shoulder.

A system of this type will undoubtedly present severe physiological problems with regard to the selection and availability of control sites. In addition there is the problem of providing control systems within the prosthesis which will give the user the best possible chance of successfully executing these more complex movements. Consider a movement of the terminal device in space, including a reorientation about the forearm axis. This may involve four separate movements. In theory these can be accomplished sequentially but there are a number of disadvantages associated with this mode of operation. Firstly, the action may look grotesque in comparison with normal movement; secondly, accurate positioning by this means can only be done by a series of successive approximations; and thirdly, and of greatest importance, it may be difficult to prevent the contents of a spoon or cup from being spilt. If one rejects 
programmed control, feeding considerations virtually eliminate sequential control as a feasible system, and the patient must learn to perform several independent motions simultaneously.

It is reasonable to suggest that closed-loop control of position as opposed to open-loop control of velocity will give the user the best chance of success. By drawing on experience in other fields it is possible to suggest further desirable qualities of the mechanical system. It is generally accepted that for power-amplifying devices controlled by a human operator the greatest ease of control is achieved if the response of the system is fast by comparison with the response of the operator. Orloff (1965) has suggested that in order to meet this requirement the dominant (or lowest) natural frequency present in the prosthetic control system should not be less than 10-15 cycles per second. With either pneumatic or hydraulic ram type actuators this frequency will almost certainly be determined by the inertial load and bulk modulus of the working fluid, and simple calculations show that frequencies of 10-15 cycles per second are unattainable with low pressure pneumatic systems.

The protagonists of pneumatic control will undoubtedly point out that man has shown himself to be remarkably adept at controlling inadequate mechanical systems. This is true, and it is indeed possible that a patient may be able to effect simultaneous control of a system with several degrees of freedom with open-loop velocity controls, but the aim should te to provide the patient with the most easily controlled prosthesis and not with a severe test of his ability to control inadequate machinery.

The higher bulk modulus of the usual fluids used in hydraulics would permit the dominant natural frequency of hydraulically operated systems to be raised to the desired order, but although hydraulic control systems are widely used in general engineering their use for prostheses will create special problems. Probably the most severe is that of energy conversion. Because of its high density and bulk modulus, hydraulic fluid is an unsuitable medium for storing energy and another must be used. The most common methods are chemical, electrical and pneumatic. Chemical storage offers the lightest source of energy but usually requires a heat engine to produce mechanical energy, and seems the least likely to be acceptable for prosthetic systems, although it would be unwise to discard the possibility of its ultimate use.

Modern rechargeable electric cells offer more energy per unit of weight than is now achieved with systems using compressed carbon dioxide, but small electric motors have to run at high speed to produce the required power and a small efficient hydraulic pump must be developed if conventional methods of conversion are to be employed.

As a short-term solution, pneumatic storage may be more suitable. Most hydraulic systems contain an accumulator to provide short bursts of energy above the capacity of the pump and it seems feasible that a small scale pneumatic-to-hydraulic converter could be incorporated with the accumulator.

The convenience and safety of the storage of electrical energy encourages serious consideration of electrically operated systems. There seems no reason why natural frequencies of the required magnitude should not be obtained. Unfortunately the necessity for high speed operation of small electric motors requires large reductions in speed between the prime mover and the output. Unless carefully designed these tend to be inefficient and to have a serious effect on the power output in relation to weight of the complete system. A further disadvantage of electric motors is the high armature current drain in the stalled condition due to the absence of any back e.m.f. Thus, unless the reduction gear is so inefficient that it is irreversible, the battery will be heavily drained if any force is exerted by the prosthesis even though it is stationary and no work is being done. Static load can be sustained without draining the battery by the use of mechanical locks but, if fully automatic, these make the system highly complex.

In addition to the engineering problems associated with raising the dominant natural frequency of the mechanical system, there are problems in connection with the complete system; that is, the man and his prosthesis viewed as a whole. The analysis of such control 


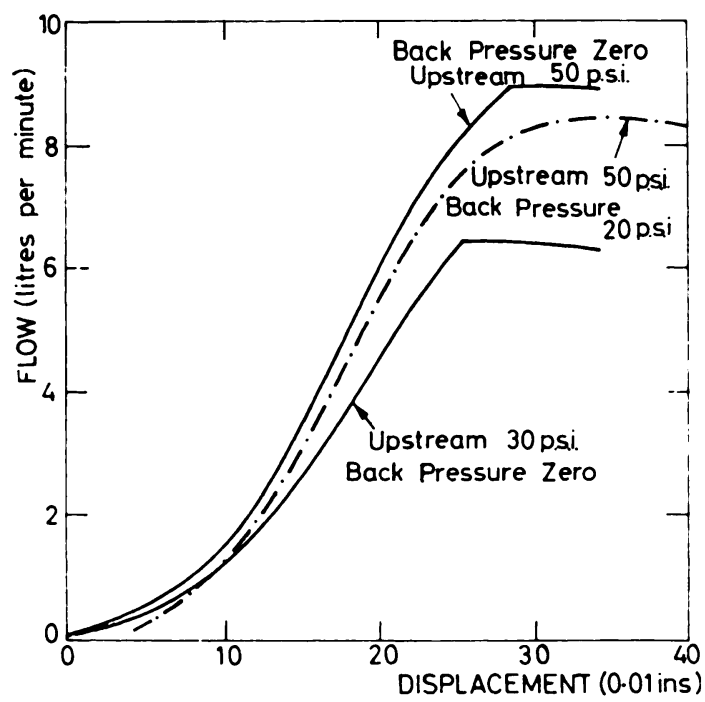

Fig. 5

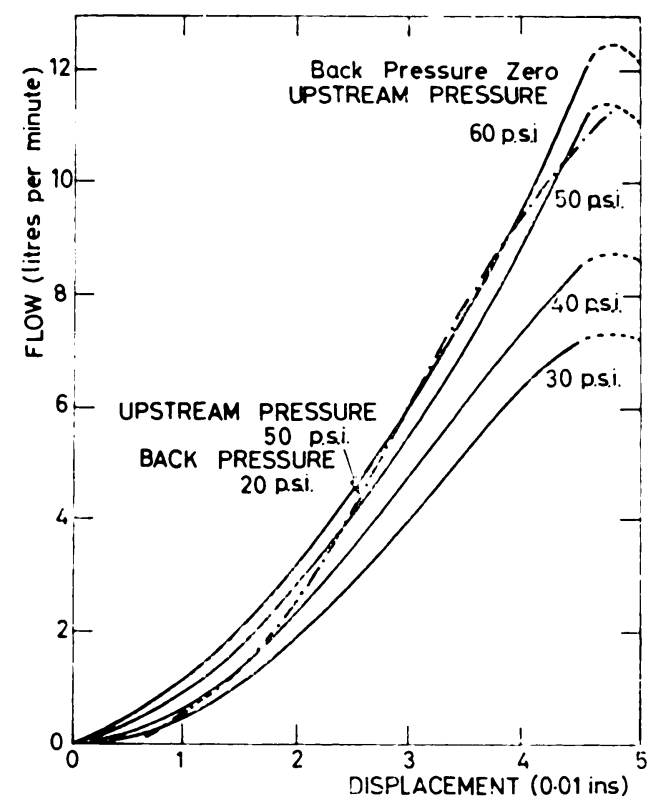

FIG. 6

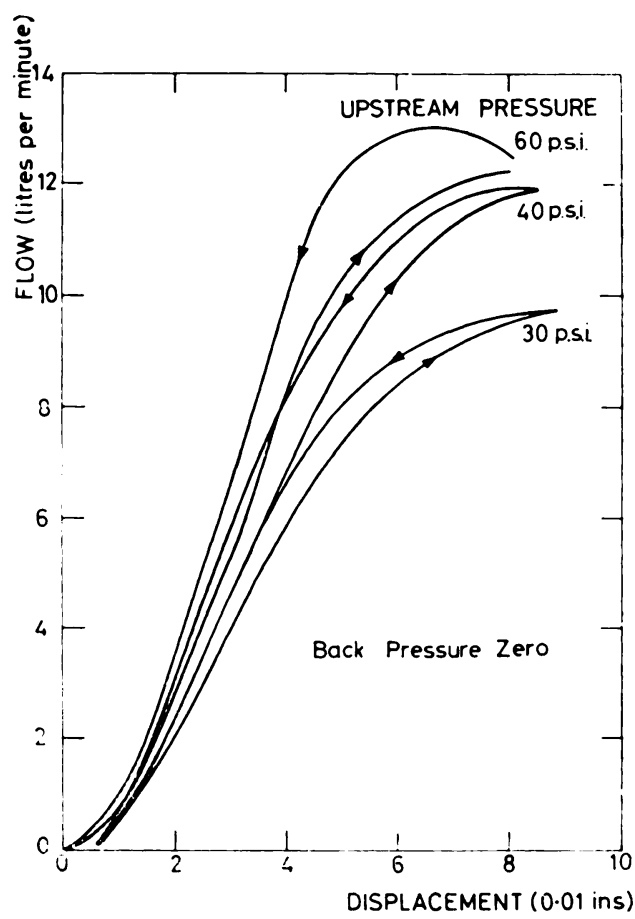

Fig. 7

Flow characteristics of control valves. Figure 5-Hendon valve. Figure 6-Kiessling valve. Figure 7Roehampton Kiessling valve. 
systems is possible if the performance of the human operator is expressed mathematically. This is difficult not only because the biological system is complex, but also because of the differences between individuals and the variation that occurs in the performance of any given individual. It seems possible that progress may be made in this analysis by the use, for example, of information theory, and this is being investigated.

Linked with this is the problem of the almost total lack of proprioceptive information associated with prostheses. This undoubtedly plays an important role in the biological system and presumably the control of multiple prostheses will be made easier if some form of proprioceptive feedback can supplement the visual link. Experiments are in progress to assess the value of different methods of feeding information back into the body. In these experiments normal subjects are required to control a simulated prosthesis with two degrees of freedom, and their performances with and without additional feedback information is compared.

The difficulty of finding a sufficient number of suitable control sites is a major factor in the rehabilitation of the severely handicapped. The selection of sites must be a matter for the clinician responsible for fitting the individual prosthesis, but it is important to make the best possible use of those sites that are available. Most of the systems in current use copy the biological system in so far that both muscles in an antagonistic pair are used to control a single movement. Experiments are being carried out in which the sum and difference of the signals from a single muscle pair are used to control two independent movements, and if these are successful the system may be worthy of consideration in cases with a severe shortage of control sites.

The research which is being carried out into multiple control systems might reasonably be described as long-term. Its results are unlikely to be fully applied for some years, and in the meantime the proven pneumatic systems must be further developed. There follows a brief description of the results of tests on the components of pneumatic systems which have been carried out by undergraduates in the Department of Mechanical Engineering of University College London.

\section{CONTROL VALVES}

Characteristics of flow versus displacement for the American Kiessling, Roehampton Kiessling and Hendon control valves have been measured and are shown in Figures 5 to 7 . For convenience the tests were carried out with compressed air, but comparable results may be expected with carbon dioxide. It may be seen that in each case the flow increases progressively but not in a straight line with lever deflection until, when the valves become fully open, no further increase of flow occurs.

The pressures quoted are measured above atmospheric with zero back pressure and with pressure ratios across the valve greater than critical. The critical pressure ratio for air is 1.89 , and if a greater pressure ratio than this occurs across a restriction sonic flow occurs at the throat and the flow depends solely upon the value of the upstream pressure and the throat area. Logarithmic plots show that for conditions of choked flow the flow characteristics of the valves can be expressed in the form $\mathrm{Q}=\mathrm{CpX} \mathrm{X}^{n}$, where $\mathrm{Q}$ is the flow, $\mathrm{p}$ the upstream pressure and $\mathrm{X}$ is the lever deflection. The coefficient $\mathrm{C}$ and the index $n$ depend upon the valve geometry.

When the pressure ratio across the valve is less than critical the flow also depends upon the downstream pressure, and this effect can be seen from the curves of upstream pressure 50 pounds per square inch and downstream pressure 20 pounds per square inch, which gives just less than the critical pressure ratio.

The Roehampton-Kiessling valve exhibits a type of hysteresis effect. For the same value of lever deflection higher flows are obtained when the valve is being closed than when it is being opened. This is thought to be due to characteristics of the rubber " $\mathrm{O}$ "-rings, and further tests are being carried out to investigate the variations that are likely to occur from manufacturing tolerances. 
PNEUMATIC VALVE FOR POSITION CONTROL BY CLOSED LOOP

A valve which is being developed for use in a laboratory model of a prosthesis with position control by closed loop is shown diagrammatically in Figure 8. For this control the valve deflection must be a function of the error between the input signal and the limb position and this is achieved as follows. Two Roehampton-Kiessling valves are mounted one above the other on a carriage, $\mathrm{C}$, which rotates above an axis through $\mathrm{A}$. The operating cable is attached to the carriage and the angle between the centre lines of the carriage and base represents the input signal, $\theta \mathrm{i}$.

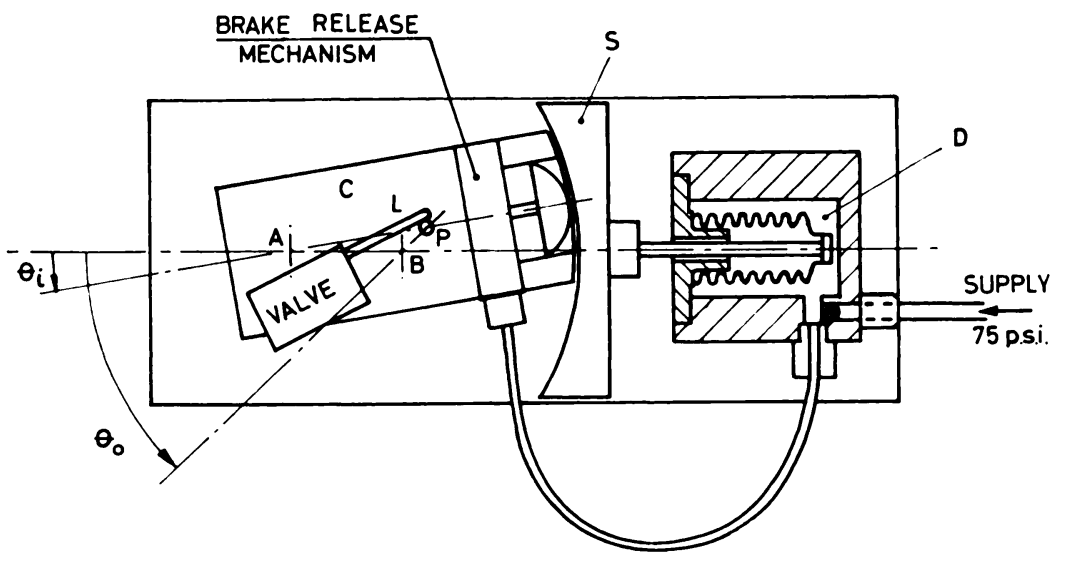

Fig. 8

Prototype valve for closed-loop position control system. The second valve is mounted directly above the first with its lever $L$ parallel to that of the first but located on the opposite side of the pin $\mathbf{P}$.

The output signal, representing the position of the prosthesis, is the angle of rotation, $\theta 0$, of the pin, $\mathrm{P}$, about an axis through $\mathrm{B}$. $\mathrm{P}$ and $\mathrm{C}$ rotate about different centres only for reasons of compactness. Thus, so long as $\theta \mathrm{i}$ and $\theta 0$ maintain a relationship such that $\mathrm{P}$ stays on the centre line of $\mathrm{C}$, there is no error and the valves remain closed. If an error exists the pin deflects one or other of the valve levers, L, so that gas flows to adjust $\theta 0$ until the error is reduced to zero.

To permit cable operation of the device the carriage has to be spring-loaded in one direction, and in order to allow the cable to be relaxed in any rest position of the prosthesis it is necessary for the carriage to be locked in the appropriate position. This is achieved by means of a brake shoe, $\mathrm{S}$, which is pushed against the carriage when supply pressure is maintained in the chamber, D. Gas is allowed to enter the chamber through an adjustable restrictor so that the filling time can be varied. The chamber is also connected to a brake release mechanism mounted in the carriage, $\mathrm{C}$. If relative movement between the carriage and brake shoe is initiated by a pull on the operating cable, a valve is deflected which opens the chamber, $\mathrm{D}$, to atmosphere and the brake is released. On completion of the movement the valve is closed and the chamber refills through the restrictor valve. The action of the device is therefore similar to that of a roller blind which can be set in any desired position and released by a small movement of the cord.

\section{ACTUATORS}

Tests have been carried out on actuators that have been used or suggested for use in gas powered systems. These were a three-quarter inch diameter single-acting piston actuator taken from a Hendon motor unit, a one and a half inch diameter metal bellows, the same bellows unit partly filled with a brass core, and a McKibben muscle. The basis for comparison was the work output from the actuator for unit mass of carbon dioxide used. The actuators were 
tested by a number of load cycles in each of which a weight representing 85 per cent of the available actuator force was raised against gravity, the actuator then being exhausted and the weight returned by gravity. The mass of carbon dioxide used was measured by weighing the storage bottle before and after each test. The results are given in Table I, which shows that

TABLE I

COMPARISON OF OUTPUT OF ACtUATORS

\begin{tabular}{|l|c|}
\hline Actuator type & $\begin{array}{c}\text { Work out } \\
\text { (foot/pounds per gramme } \\
\text { carbon dioxide) }\end{array}$ \\
\hline Piston .. & 15.4 \\
Bellows with internal core & 8.7 \\
Bellows (hollow) . & 6.3 \\
McKibben muscle . & 5.0 \\
\hline
\end{tabular}

the piston actuator is capable of giving the greatest economy in gas. It should be noted, however, that the form of test favoured the piston actuator in that the load cycle was always started at the bottom of the piston stroke when the initial volume was practically zero. In prosthetic use, where the actuator may be started from any point in its stroke with only

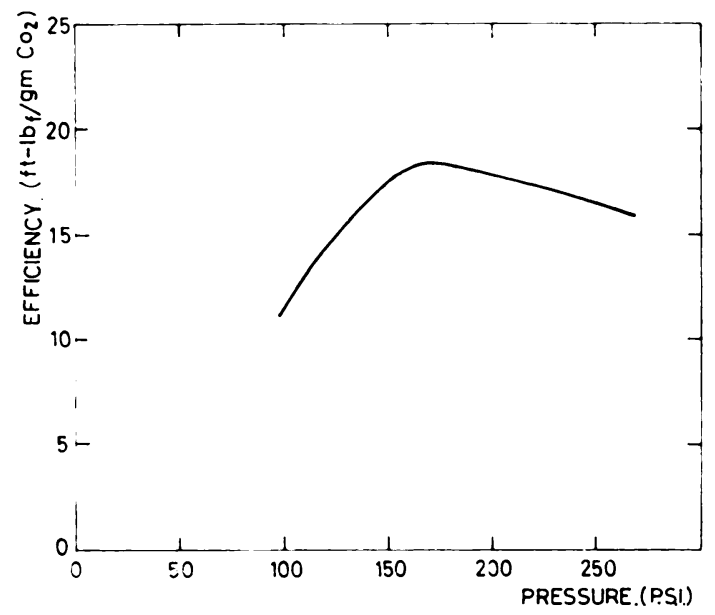

FIG. 9

Variation with working pressure of the work output per gramme of carbon dioxide used.

partial compression of the initial volume, a lower output must be expected: nevertheless, it seems likely that the piston actuator would still compare favourably with the other types tested.

In fairness to the McKibben muscle it should be stated that this was of an early type, and it is possible that later designs may show improved efficiency.

\section{WORKING PRESSURE}

At present most British systems use a working pressure in the region of 70-100 pounds per square inch. If the work output per unit mass of carbon dioxide is taken as a criterion, theoretical analysis shows that the optimum working pressure is in the region of 200-450 pounds 
per square inch. The exact optimum is difficult to define, because it depends upon the amount of reheating of the gas after throttling at the reducing valve (the temperature of the gas immediately after constant enthalpy throttling from 750 to 250 pounds per square inch is about -23 degrees Centigrade), and also upon the amount of further expansion that occurs in the actuator cylinder.

The results of tests at higher working pressures, shown in Figure 9, support the theoretical findings and show that significant savings of gas could be achieved if pressures were raised to 150-200 pounds per square inch. An additional benefit to be obtained from this increase would be the reduction in actuator weight from the use of smaller piston diameters.

The views expressed in this paper are not those of the author alone but represent the results of much discussion with his colleagues, Dr R. M. Davies and Mr M. J. Hall. The results of the tests on control valves and actuators for pneumatic systems were obtained as part of their final year project by four undergraduates, Mr I. D. Burnett, Mr A. M. Davies, Mr P. A. Kirkwood and Mr R. J. G. Price.

In this difficult interdisciplinary field it is impossible to work efficiently in isolation from the clinical problems. The author and his colleagues gratefully acknowledge the help they have received from the staffs of the Centre for Muscle Substitutes, Hendon, and the Research Department of the Limb Fitting Centre at Roehampton.

\section{REFERENCE}

Orloff, G. (1965): Multiple Prosthetic Systems, Fluid Dynamics-Technical Note No. 6, Mollins Machine Co. Ltd. 